Note

\title{
Differential effects of mitogen-activated protein kinase pathway inhibitors on P-glycoprotein activation
}

\author{
Hirokazu Wakuda ${ }^{1, *}$, Shino Miyauchi ${ }^{1}$, Kana Maruyama ${ }^{1}$, Satomi Kagota ${ }^{1}$, Kazuki \\ Nakamura ${ }^{1}$, Keizo Umegaki ${ }^{2}$, Shizuo Yamada ${ }^{3}$ and Kazumasa Shinozuka ${ }^{1}$ \\ ${ }^{1}$ Department of Pharmacology, School of Pharmacy and Pharmaceutical Sciences, Mukogawa Women's University, 11- \\ 68 Koshien, Kyuban-cho, Nishinomiya 663-8179, Japan \\ ${ }^{2}$ Information Center, National Institute of Health and Nutrition, 1-23-1 Toyama, Shinjuku-ku, Tokyo 162-8636, Japan \\ ${ }^{3}$ Center for Pharma-Food Research (CPFR), Graduate School of Pharmaceutical Sciences, University of Shizuoka, 52-1 \\ Yada, Suruga-ku, Shizuoka, Shizuoka 422-8526, Japan
}

*Corresponding Author: E-mail: wakuda@mukogawa-u.ac.jp; Tel.: +81-798-45-9944; Fax: +81-798-45-9944

Received: November 12, 2014; Revised: March 17, 2015; Published: March 31, 2015

\begin{abstract}
The aim of this study was to evaluate the effects of the mitogen-activated protein kinase (MAPK) pathway inhibitors SB203580, CMPD-1, SB239063, SP600125, and FR180204 on the activity of P-glycoprotein (P-gp) and to assess whether the MAPK pathway affects $P$-gp directly. Changes in the fluorescence of residual rhodamine 123, a marker of P-gp activity, in the apical region of Caco-2 cells were measured in the presence of MAPK pathway inhibitors using time-lapse confocal laser scanning microscopy at $0,10,20,30$, and $60 \mathrm{~min}$. Significant differences were observed between the fluorescence levels of control cells and cells treated with SB203580 for 20, 30, or $60 \mathrm{~min}$. However, no significant change was observed in the residual rhodamine 123 fluorescence of cells treated with CMPD-1, SB239063, SP600125, or FR180204. Among the p38-MAPK pathway inhibitors investigated, CMPD-1 and SB239063 showed no detectable effect on the activity of P-gp. Further, JNK 1, 2, 3-MAPK pathway (SP600125) and ERK1/2 pathway (FR180204) inhibitors did not affect $P$-gp activity. However, SB203580 enhanced the transfer of rhodamine 123 across the apical cell membrane. Thus, SB203580 activated P-gp, although not through the p38-MAPK pathway. Importantly, the MAPK pathway did not affect P-gp activity shortly after treatment.
\end{abstract}

\section{Keywords}

Confocal laser scanning microscopy; MAPK; SB203580

\section{Introduction}

P-glycoprotein (P-gp) is the most thoroughly studied member of the adenosine triphosphate-binding cassette transporter superfamily and is expressed throughout the intestinal epithelium, hepatocytes, renal tubular cells, and the blood-brain barrier [1]. P-gp is encoded by the ABCB1/MDR1 gene in humans [2]. Because of its ubiquitous expression and broad specificity, changes in P-gp expression or efflux activity induced by drug treatments, diet, environmental factors, or single nucleotide polymorphisms (SNPs) can greatly affect drug disposition, pharmacokinetics, and clinical response [3]. Hence, identification of P-gp 


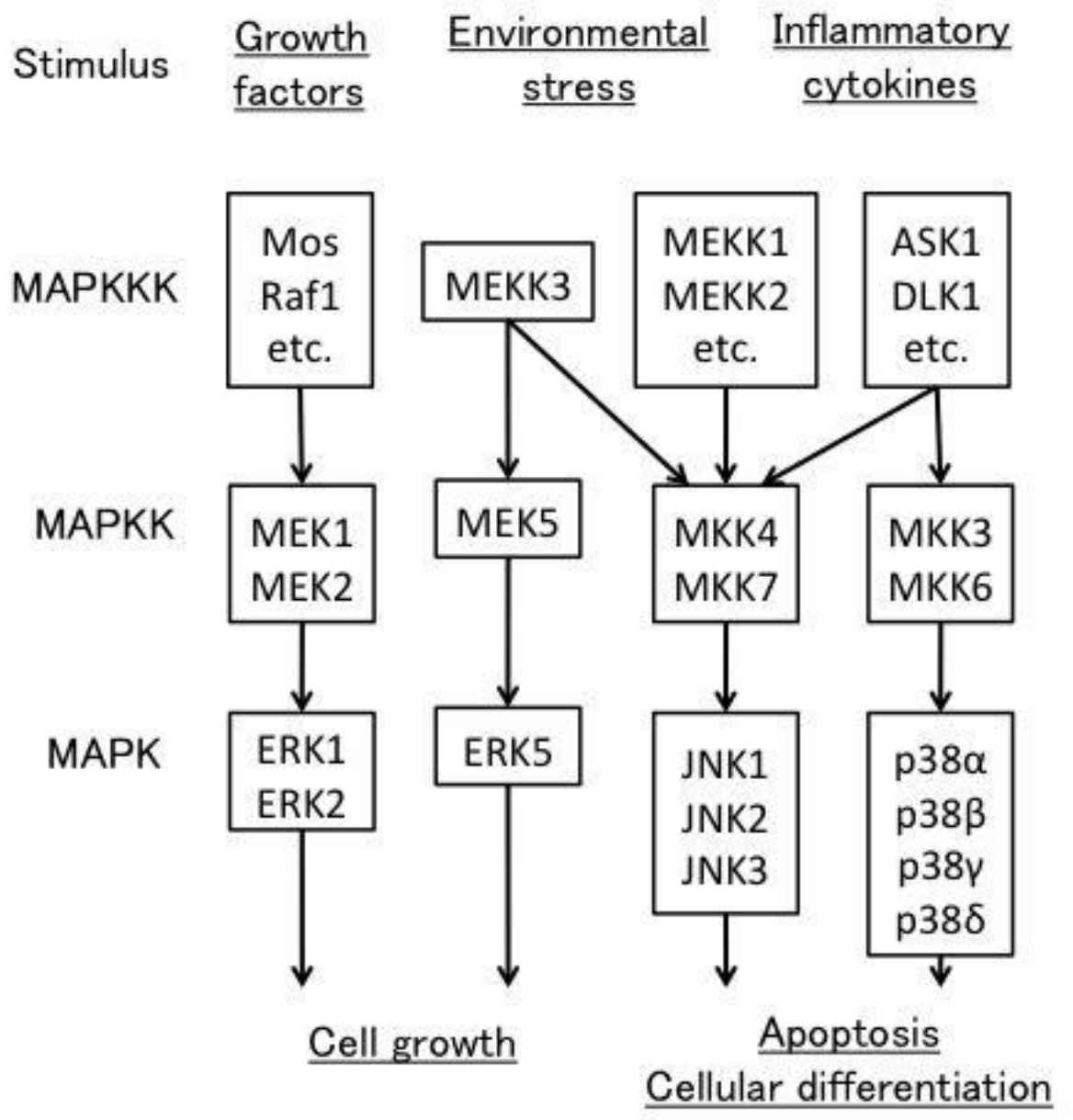

Figure 1. The MAPK pathway

substrates is important for therapeutic optimization and to avoid drug-drug interactions. However, little is known of P-gp regulation.

Mitogen-activated protein kinases (MAPKs) are a family of Ser/Thr protein kinases that are widely conserved among eukaryotes and are involved in many cellular functions. The p38-MAPK and c-Jun Nterminal kinase (JNK)-MAPK signalling pathways allow cells to interpret a wide range of external signals and respond appropriately by generating a plethora of different biological effects. There are four p38 subtypes (p38 $\alpha, p 38 \beta, p 38 \gamma, p 38 \delta)$ and three JNK subtypes (JNK1, JNK2, JNK3) [4,5]. The extracellular signalregulated kinase (Erk1/2)-MAPK signalling pathway can be activated in response to a diverse range of extracellular stimuli, including mitogens, growth factors, and cytokines (Fig. 1) [6]. Several studies have indicated that MAPK signalling pathways may regulate membrane trafficking proteins. For example, Fujishiro et al. reported that activation of the p38-MAPK pathway is not necessary for insulin-induced glucose uptake but that it regulates glucose transporter expression [7]. Nagelin et al. reported that murine 12/15-lipoxygenase regulates the expression and function of the ATP-binding cassette transporter G1 through p38- and JNK2-dependent pathways [8]. These reports suggest that the MAPK pathway transmits signals to membrane trafficking proteins to regulate their activity.

In the present study, we evaluated the effects of MAPK pathway inhibitors (SB203580, competitive p38 $\alpha$ and $\beta$ inhibitor; CMPD-1, non-competitive p38 $\alpha$ inhibitor; SB239063, competitive $p 38 \alpha$ and $\beta$ specific inhibitor; SP600125, competitive JNK 1, 2, 3 inhibitor; FR180204, competitive ERK1/2 inhibitor) on the 
activity of P-gp using time-lapse confocal laser scanning microscopy. The confocal laser scanning microscopy-based assay was used to measure residual rhodamine 123 in the apical region of Caco-2 cells; this method is more sensitive than conventional methods that rely on microplate readers and fluorescence microscopy $[9,10]$.

\section{Experimental}

\section{Materials}

SB203580 was obtained from InvivoGen (San Diego, CA, U.S.A.). CMPD-1 was obtained from Tocris Bioscience (Ellisville, MO, U.S.A.). SB239063 was obtained from Sigma-Aldrich (St. Louis, MO, U.S.A.). SP600125 was obtained from Cayman Chemical (Ann Arbor, MI, U.S.A.). FR180204 was obtained from Santa Cruz Biotechnology (Santa Cruz, CA, U.S.A.). Rhodamine 123 was obtained from Molecular Probes (Eugene, OR, U.S.A.).

\section{Cell culture of Caco-2 cells}

The human colon adenocarcinoma cell line (Caco-2) was purchased from DS Pharma Biomedical (Osaka, Japan). The cells were used for experiments $7 \mathrm{~d}$ after seeding and between passages 47 and 52 .

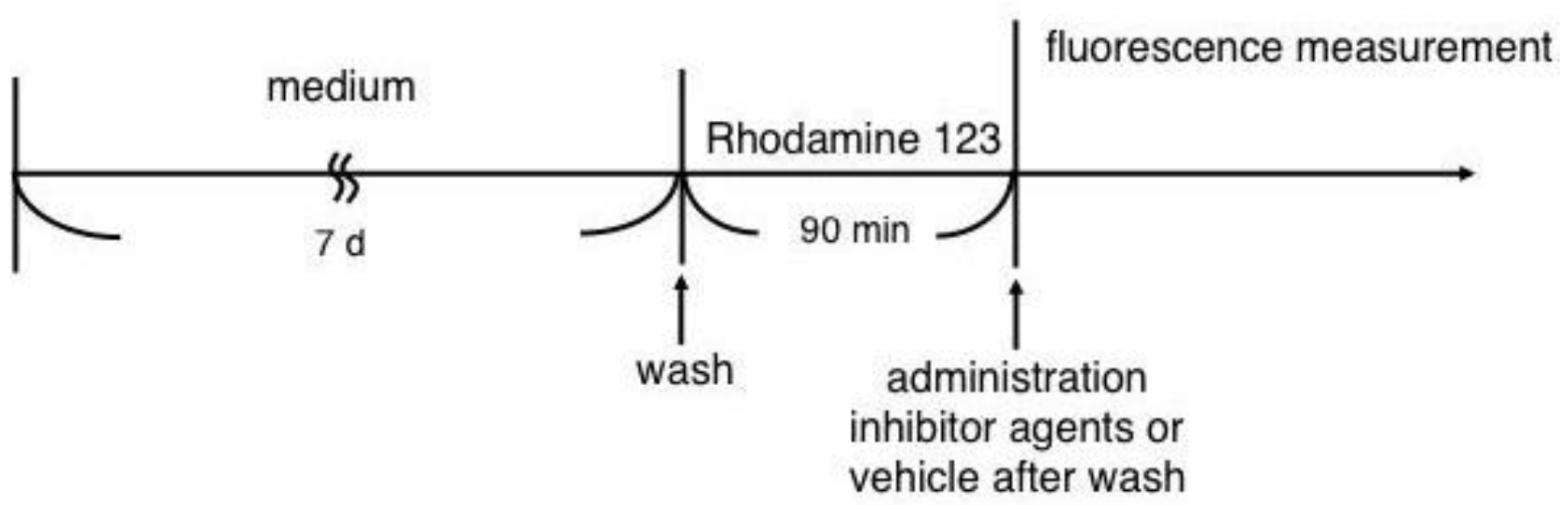

Figure 2. The protocol for measurement of P-glycoprotein-mediated transport activity using confocal laser-scanning microscopy in the presence or absence of inhibitor agents.

\section{Quantification of rhodamine 123 by time-lapse confocal laser scanning microscopy}

Transport measurements were performed in a transwell chamber (BD Biosciences, San Jose, CA, U.S.A.). Caco- 2 cells were seeded at a density of $3 \times 104$ cells per filter on polyethylene terephthalate filters in the cell culture inserts.

The cell culture inserts were placed on a glass-bottomed dish, and the Caco-2 cells were incubated with $5 \mu \mathrm{M}$ rhodamine 123 for $90 \mathrm{~min}$ at $37^{\circ} \mathrm{C}$. The cells were rinsed with Hanks' Balanced Salt Solution (HBSS). HBSS with or without MAPK pathway inhibitors was added to the cells (SB203580, $10 \mu \mathrm{M}$; CMPD-1, $10 \mu \mathrm{M}$; SB239063, 100 nM; SP600125, 100 nM; FR180204, 1 MM), and cells were observed by time-lapse confocal laser scanning microscopy (Fig. 2). Quantification of the intracellular concentration of rhodamine 123 in the 
Caco- 2 cells was performed using fluorescent images at several time points $(0,10,20,30$, and 60 min) by a chronological measurement program (EZ-C1; Nikon, Tokyo, Japan). The fluorescence intensity at 0 min was defined as $100 \%[9,10]$.

\section{Statistical analysis}

All values represent the mean \pm standard error. The data were analysed using a two-way ANOVA followed by a Bonferroni post-test to determine significance $(P<0.05)$. Statistical analyses were performed using the GraphPad Prism 4.03 software (GraphPad Software, La Jolla, CA, U.S.A.).

\section{Results and Discussion}

The level of residual rhodamine 123 fluorescence in control cells and cells treated with MAPK pathway inhibitors were measured with time-lapse confocal laser scanning microscopy to assess P-gp activity. Treatment with $10 \mu \mathrm{M}$ SB203580, a competitive inhibitor of the p38 $\alpha$ and $\beta$ ATP binding pocket, enhanced the fluorescent decrease significantly at 20, 30, and $60 \mathrm{~min}$ (Fig. 3a). In contrast, the decrease in fluorescent intensity was not affected by treatment with the JNK 1, 2, 3 inhibitor, SP600125 (100 nM), or the ERK1/2 inhibitor FR180204 (1 $\mu \mathrm{M})$ (Fig. 3b, c). Further, the decrease in fluorescent intensity was not affected by the non-competitive p38 $\alpha$ inhibitor CMPD-1 $(10 \mu \mathrm{M})$ (Fig. 3d) or a second, more specific competitive inhibitor of the p38 $\alpha$ and $\beta$ ATP binding pocket, SB239063 (100 nM) (Fig. 3e).

We evaluated the effects of MAPK pathway inhibitors on the activity of P-gp, using time-lapse confocal laser scanning microscopy. SB203580 enhanced the fluorescent decrease significantly after $20 \mathrm{~min}$. It is hypothesized that the enhanced effect of SB203580 is due to changes in P-gp activation, as changes to the P-gp expression level would require at least $24 \mathrm{~h}$ of drug treatment. SB203580 is a pyridinyl imidazole that competitively inhibits ATP binding and is widely used in studies for elucidating the roles of p38-MAPK [11]. Barancík et al. reported that treatment of the multidrug resistant mouse leukaemia cell line L1210/VCR with SB203580 (30 $\mu \mathrm{M})$ for 3 days inhibited P-gp [12]. In contrast, our results suggest that treatment of the human colon adenocarcinoma cell line Caco-2 with SB203580 (10 $\mu \mathrm{M})$ for 60 min activates P-gp. The difference in effect may be due to differences in cell lines, SB203580 concentration, and duration of treatment.

Furthermore, the decrease in fluorescent intensity was not affected by SP600125 and FR180204. SP600125 is a JNK 1, 2, 3 inhibitor and FR180204 is an ERK1/2 inhibitor. Our results suggest that the JNKMAPK and ERK1/2-MAPK pathways do not affect P-gp activity directly or over the time course studied. Supporting our data, Kim et al. have also reported that SP600125 had no effect on P-gp [13].

The enhancement of P-gp activity following SB203580 treatment suggested that activation of the p38MAPK pathway might inhibit P-gp. As mentioned earlier, SB203580 competitively inhibits ATP binding to p38 $\alpha$ and $\beta$. Further, P-gp is an ATP-dependent transporter. Thus, we hypothesized that inhibition of ATP binding to $p 38 \alpha$ and $\beta$ by SB203580 might enhance available ATP levels, thereby enhancing the activation of P-gp. To further assess the contribution of p38-MAPK pathways, cells were treated with either CMPD-1 or SB239063. CMPD-1 is a selective, non-competitive inhibitor of $p 38 \alpha$, and SB239063 is a competitive inhibitor of p38-MAPK [14,15]. Importantly, SB239063 is more specific than SB203580 [15]. Interestingly, both CMPD-1 and SB239063 had no detectable effect on the activity of P-gp. Thus, our results suggest that 
the p38-MAPK pathway does not affect P-gp directly after a short duration of treatment. However, SB203580 increased the transfer of rhodamine 123 across the cell membrane after $20 \mathrm{~min}$.
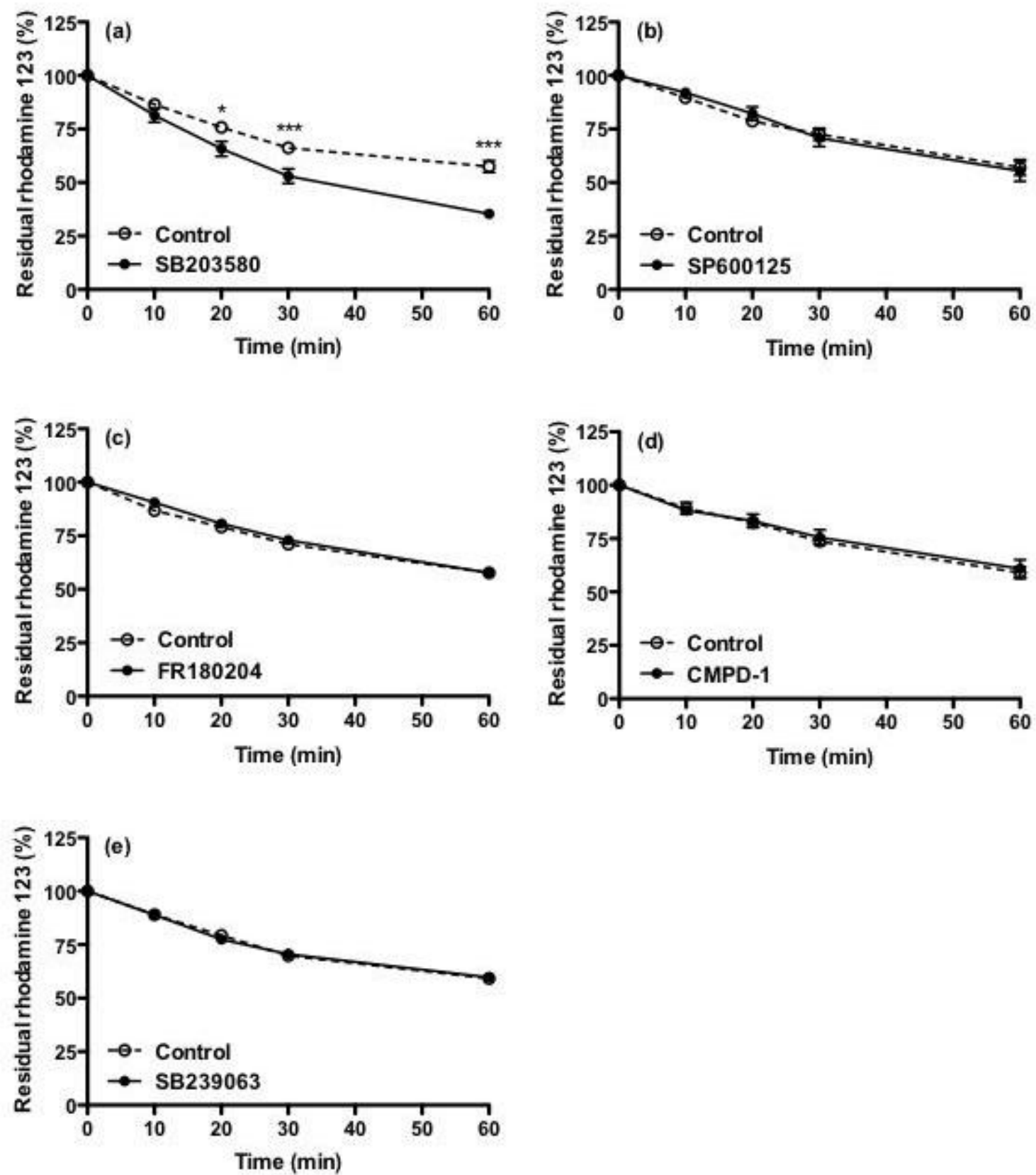

Figure 3. Influence of MAPK pathway inhibitors on the time-dependent decrease in residual rhodamine 123 fluorescence in the apical region of Caco-2 cells, measured by time-lapse confocal laser scanning microscopy. Each chamber represents a p38-MAPK pathway inhibitor: (a) SB203580, $10 \mu \mathrm{M}$ : (b) SP600125, 100 nM; (c) FR180204, $1 \mu$ M: (d) CMPD-1, $10 \mu \mathrm{M}$ : (e) SB239063, $100 \mathrm{nM}$. Each point and bar represents the mean \pm standard error. $\mathrm{n}=12-18$. Significant difference between the control group and SB203580 group $(P<0.05,0.001)$.

There are two possible reasons for the enhanced rhodamine transport. Firstly, the effect of SB203580 could be mediated through pathways other than the p38-MAPK pathway. The working concentration of SB203580 reported in the literature is 1-20 $\mu \mathrm{M}$. However, at high concentrations, SB203580 can also affect Raf-1, phosphoinositide-dependent protein kinase 1 (PDK1), the transforming growth factor-beta (TGF- $\beta$ ) receptor, cyclooxygenase 1 (COX-1), and cyclooxygenase 2 (COX-2) [16]. Additionally, Lali et al. reported that low concentrations of SB203580 can inhibit the phosphorylation and activation of protein kinase B 
(PKB), also known as Akt, by inhibiting the PKB kinase, PDK1 (IC50, 3-5 $\mu \mathrm{M}$ ) [17]. It is possible that the activation of P-gp by SB203580 may occur via one of these pathways. Alternatively, SB203580 could cause a structural change in P-gp. P-gp is one of the best-characterized ABC transporters; however, the mechanism of substrate transport is unclear. In particular, it has been hypothesized that there are many possible drug-binding sites on P-gp, including up to seven putative binding sites located in the transmembrane domain of the protein [18-20]. It is generally accepted that transmembrane helices 5, 6, 11, and 12 are involved in P-gp substrate binding. One popular model published by Shapiro et al. suggested that two different functional binding sites, the $\mathrm{R}$-site and $\mathrm{H}$-site, interact in a positive cooperative manner.18 Rhodamine 123 and anthracylines are substrates of the R-site, whereas colchicine is a substrate of the $\mathrm{H}$-site of P-gp. This two-site hypothesis is the most convenient working model that explains the mutual stimulation of P-gp-mediated transport by several substrates. Additionally, there is evidence of a third allosteric binding site that serves a regulatory function and may be the site of progesterone binding [21]. Sterz et al. reported that some substances are activators of P-gp [22]. Further, Kerboeuf et al. reported that macrocyclic lactone anthelmintics activate P-gp in nematodes and suggested that several substituents in the macrocyclic lactone structure are involved in modulating P-gp activation [23].

\section{Conclusions}

The MAPK pathway does not affect short-term P-gp activation. Rather, activation of P-gp by SB203580 is likely due to either pathways other than the p38-MAPK pathway or allosteric structural changes in P-gp. Thus, further studies should be performed to understand the mechanism underlying SB203580-mediated P-gp activation.

Acknowledgements: The authors declare that they have no conflicts of interest to disclose and funding. The authors are grateful to Prof. Yoshiyuki Kagawa, Prof. Junko Sugatani and Prof. Hiroshi Yamada for their suggestions. We also thank our colleagues for their helpful advice and support.

\section{References}

[1] F. Thiebaut, T. Tsuruo, H. Hamada, MM. Gottesman, I. Pastan, MC. Willingham, Proc. Natl. Acad. Sci. USA. 84 (1987) 7735-7738.

[2] VJ. Wacher, CY. Wu, LZ. Benet, Mol. Carcinog. 13 (2000) 129-134.

[3] M. Hitzl, S. Drescher, H. van der Kuip, E. Schäffeler, J. Fischer, M. Schwab, M. Eichelbaum, MF. Fromm, Pharmacogenetics 11 (2001) 293-298.

[4] A. Cuadrado, AR Nebreda, Biochem. J. 429 (2010) 403-417.

[5] MA. Bogoyevitch, KR. Ngoei, TT. Zhao, YY. Yeap, DC. Ng, Biochim. Biophys. Acta. 1804 (2010) 463475.

[6] S. Nishimoto, E. Nishida, EMBO Rep. 7 (2006) 782-786.

[7] M. Fujishiro, Y. Gotoh, H. Katagiri, H. Sakoda, T. Ogihara, M. Anai, Y. Onishi, H. Ono, M. Funaki, K. Inukai, Y. Fukushima, M. Kikuchi, Y. Oka, T. Asano, J. Biol. Chem. 276 (2001) 19800-19806.

[8] MH. Nagelin, S. Srinivasan, JL. Nadler, CC. Hedrick, J. Biol. Chem. 284 (2009) 31303-31314.

[9] H. Wakuda, N. Nejime, Y. Tada, S. Kagota, K. Umegaki, S. Yamada, K. Shinozuka, Biol. Pharma. Bull. 33 (2010) 1238-1241.

[10] H. Wakuda, N. Nejime, Y. Tada, S. Kagota, OA. Fahmi, K. Umegaki, S. Yamada, K. Shinozuka. J. Pharm. Pharmacol. 63 (2011) 1015-1021. 
[11] A. Cuenda, J. Rouse, YN. Doza, R. Meier, P. Cohen, TF. Gallagher, PR. Young, JC. Lee, FEBS Lett. 364 (1995) 229-233.

[12] M. Barancík, V. Bohácová, J. Kvackajová, S. Hudecová, O. Krizanová, A. Breier, Eur. J. Pharm. Sci. 14 (2001) 29-36.

[13] JH. Kim, M. Chae, AR. Choi, H. Sik Kim, S. Yoon, Eur. J. Pharmacol. 723 (2014) 141-147.

[14] W. Davidson, L. Frego, GW. Peet, RR. Kroe, ME. Labadia, SM. Lukas, RJ. Snow, S. Jakes, CA. Grygon, C. Pargellis, BG. Werneburg, Biochemistry 43 (2004) 11658-11671.

[15] DC. Underwood, RR. Osborn, CJ. Kotzer, JL. Adams, JC. Lee, EF. Webb, DC. Carpenter, S. Bochnowicz, HC. Thomas, DW. Hay, DE. Griswold, J. Pharmacol. Exp. Ther. 293 (2000) 281-288.

[16] Y. Zhang Y, J. Zhou, W. Xu, A. Li, J. Zhou, S. Xu, J. Toxicol. Environ. Health. A. 72 (2009) 774-781.

[17] FV. Lali, AE. Hunt, SJ. Turner, BM. Foxwell, J. Biol. Chem. 275 (2000) 7395-7402.

[18] AB. Shapiro, V. Ling, Biochem. Pharmacol. 53 (1997) 587-596.

[19] C. Martin, G. Berridge, CF. Higgins, P. Mistry, P. Charlton, R. Callaghan, Mol. Pharmacol. 58 (2000) 624-632.

[20] AR. Safa, Curr. Med. Chem. Anticancer Agents. 4 (2004) 1-17.

[21] AB. Shapiro, K. Fox, P. Lam, V. Ling, Eur. J. Biochem. 259 (1999) 841-850.

[22] K. Sterz, L. Möllmann, A. Jacobs, D. Baumert, M. Wiese, ChemMedChem 4 (2009) 1897-1911.

[23] D. Kerboeuf, F. Guégnard, Antimicrob. Agents Chemother. 55 (2011) 2224-2232. 